\title{
Surgical Managemet of Dystocia in Snakes and Lizards
}

\author{
Lucia BEL ${ }^{*}$, Andrei MIHALCA, Cosmin PEŞTEAN, Ciprian OBER, Liviu OANA \\ Faculty of Veterinary Medicine,University of Agricultural Sciences and Veterinary Medicine Cluj- \\ Napoca, CaleaManastur nr. 3-5, 400035 ClujNapoca, Romania. \\ Corresponding author: lucia.g.bel@gmail.com
}

Bulletin UASVM Veterinary Medicine 72(1) / 2015,

Print ISSN 1843-5270; Electronic ISSN 1843-5378

DOI:10.15835/buasvmcn-vm: 11107

\begin{abstract}
Dystocia is one of the most common disorders in reptiles. Regardless of the cause of this reproductive tract disorder, in most of the cases, surgery is the option of treatment. In female chameleons this is a common disorder mostly due to the environmental conditions and very young age of reproduction.

Four female reptiles, three chameleons (Chamaeleo caliptratus) and a snake (Orthriophis taeniurus taeniurus) were presented to our clinic and diagnosed with dystocia. After the females were anesthetized using Butorphanol, Propofol and Isoflurane, coeliotomy was performed. After exploration of the coelom ovariosalpingotomy through a paramedian coeliotomy was performed in the case of the female chameleons and salpingotomy with eggs extraction in the case of the female snake, through a latero-costal coeliotomy. The salpinx was closed using a continous suture and a Cushing continous pattern (monofilament absorbable 4.0) and the coelom, in all cases, using 5.0 monofilament absorbable material. The skin was closed in an everted pattern using 4.0 monofilament nonabsorbable material. All four animals recovered well after surgery and received postoperative treatment, antibiotics (Cefuroxime, $20 \mathrm{mg} / \mathrm{kg}$ ) for 10 to 20 days and analgesia using Meloxicam (0.2 mg/kg, every 48 hours). The suture material was removed 1 month after the surgical procedures.
\end{abstract}

Keywords: dystocia, lizards, snakes,surgical management.

\section{INTRODUCTION}

A relatively common disorder in reptiles, dystocia is caused by the inability of the eggs to pass through the oviduct and cloaca, occurring most often in first time breeding females, females who have previously retained eggs or females bearing infertile clutches (Mader D, 1995). In snakes and lizards the visible appearance of swelling caused by the mass of eggs is clue to their presence. If there is no indication of infection, metabolic bone disease or obstruction, conservative treatment should be attempted, providing of a suitable environment and nesting site or medical treatment with oxytocin (Divers, 1996). In most of the cases, surgical management is mandatory.

\section{AIMS AND OBJECTIVES}

The objective of this paper was to describe the surgical management of dystocia in four reptiles presented for treatment and diagnosis in our clinic.

\section{MATERIALS AND METHODS}

Three female chameleons (Chamaeleo caliptratus) and a Chinese beauty rat snake (Orthriophis taeniurus taeniurus) diagnosed with dystocia were operated in our department. Prior to the surgical procedure, fluid therapy was initiated, by placing an intraoseous catheter in the hindleg of the chameleons and an intravenous catheter in the right jugular vein of the snake, after denudation.

The anesthetic protocol consisted of the administration of Butorphanol $(0.4 \mathrm{mg} / \mathrm{kg})$ IM 30 minutes prior to the administration of Propofol $(10 \mathrm{mg} / \mathrm{kg}), \mathrm{IV}$ in the ventral coccygeal vein. After intubation, Isoflurane $3 \%$ was used for maintenance. 

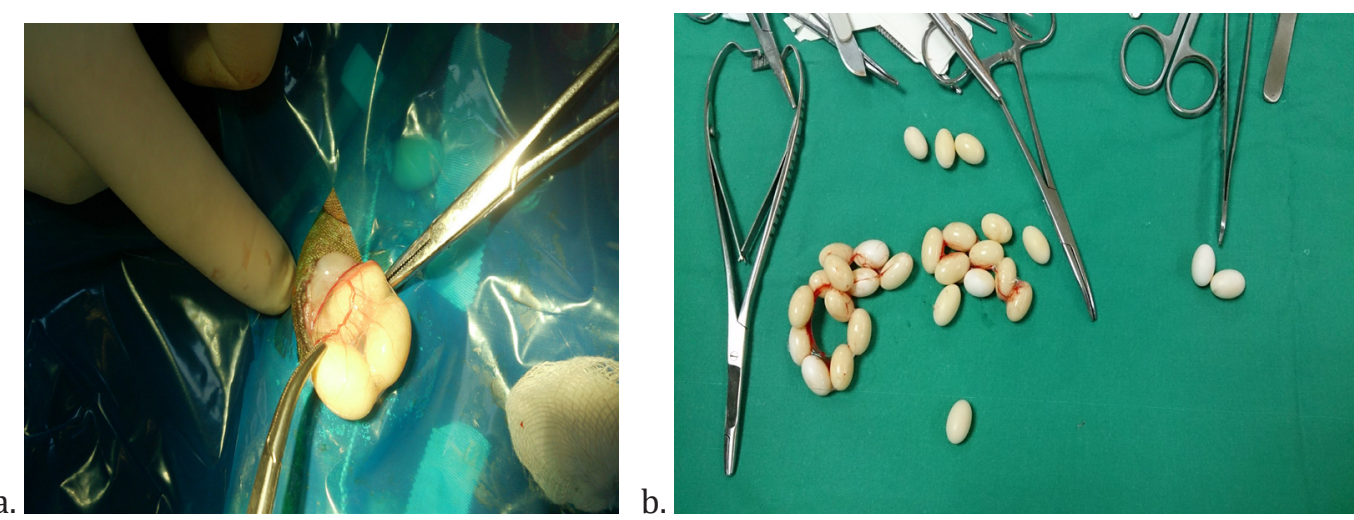

Fig. 1. (a) Isolation of the eggs in ovariosalpingectomy of female chameleons; (b) Eggs removed.
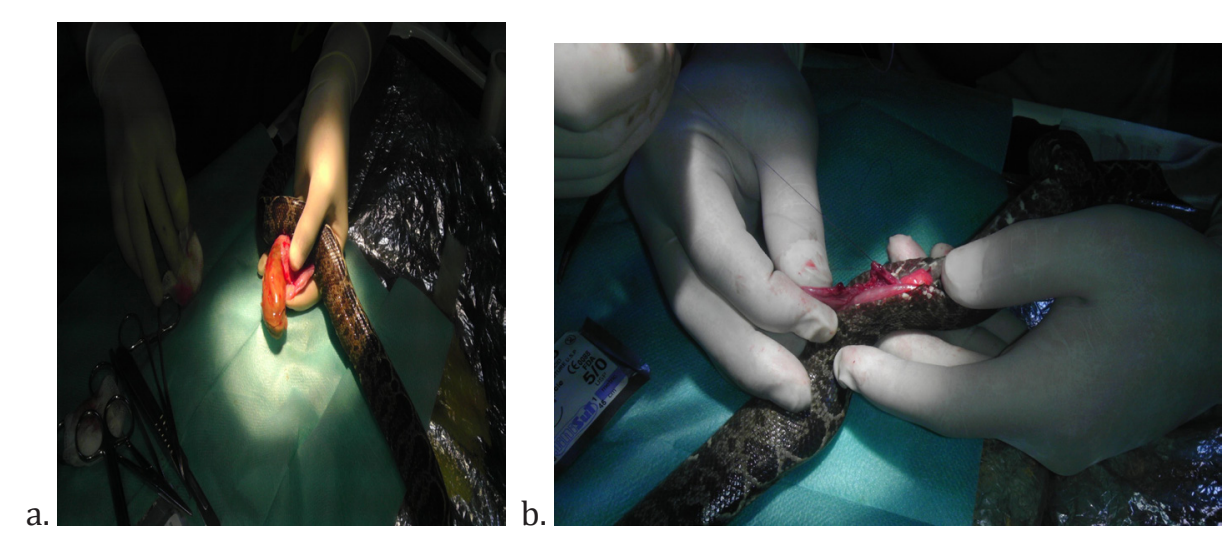

Fig. 2. (a) Salpingotomy and removal of the egg in; (b) Eggs removed.

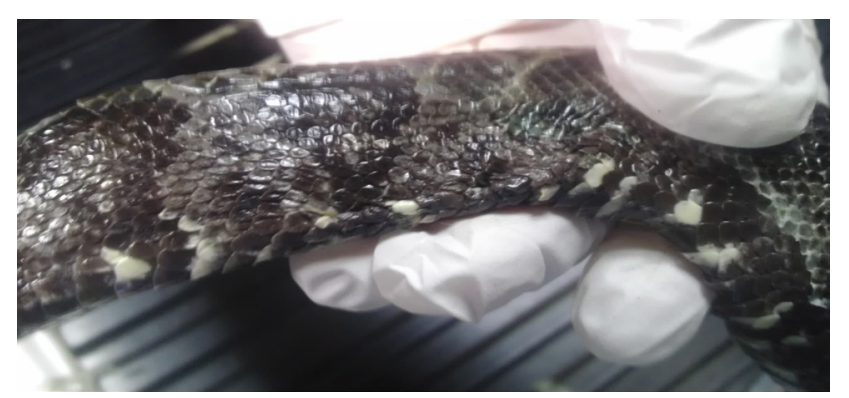

Fig.3. Skin suture of the female snake one month after surgery.

In the case of the chameleons, the skin incision was performed lateral to the abdominal vein, in the case of the snake, at the border of the rib skales and the abdominal ones. After evaluation of the coelomic cavity ovariosalpingectomy was performed in all three chameleons and salpingotomy, followed by egg extraction in the snake. Suture materials used were monofilament absorbable materials (5.0 and 4.0) for the coelomic cavity, the skin was closed using a vertical pattern with monofilament non absorbable (4.0).

\section{RESULTS}

All the animals recovered after surgery, fluid therapy was continued for one week, analgesia was achieved using Meloxicam $(0.2 \mathrm{mg} / \mathrm{kg})$ and antibiotic therapy was mandatory (Cefuroxime, 20 $\mathrm{mg} / \mathrm{kg}$ ) for 10 to 20 days after.

\section{CONCLUSION}

Surgical management of dystocia in reptiles is sometimes the only solution for saving these animals. The risk of infection increases with time, meaning that the surgical procedure should be done as soon as possible after other attempts of inducing laying have failed.

\section{REFERENCES}

1. Dyvers SJ (1996). Medical and surgical treatment of preovulatory ova stasis and postovulatory egg stasis in oviparous lizards. Proc ARAV 119-123, 24-27, Florida.

2. Mader D (1995). Reptile Medicine and Surgery, 370-374, W.B. Saunders, Philadelphia. 